\title{
Superposition Principle and the Problem of the Additivity of the Energies and Momenta of Distinct Electromagnetic Fields
}

\author{
Eduardo Notte-Cuello ${ }^{(1)}$ and Waldyr A. Rodrigues Jr. ${ }^{(2)}$ \\ ${ }^{(1)}$ Departamento de Matemáticas, \\ Universidad de La Serena \\ Av. Cisternas 1200, La Serena-Chile

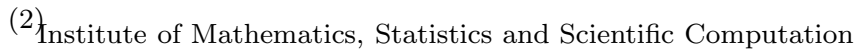 \\ IMECC-UNICAMP CP 6065 \\ 13083-859 Campinas, SP, Brazil \\ e-mail: enotte@userena.cl and walrod@ime.unicamp.br
}

November 7, 2018

\begin{abstract}
In this paper we prove in a rigorous mathematical way (using the Clifford bundle formalism) that the energies and momenta of two distinct and arbitrary free Maxwell fields (of finite energies and momenta) that are superposed are additive and thus that there is no incompatibility between the principle of superposition of fields and the principle of energy-momentum conservation, contrary to some recent claims. Our proof depends on a noticeable formula for the energy-momentum densities, namely, Riesz formula $\star \mathcal{T}^{\mathbf{a}}=\frac{1}{2} \star\left(F \theta^{\mathbf{a}} \tilde{F}\right)$, which is valid for any electromagnetic field configuration $F$ satisfying Maxwell equation $\partial F=0$.
\end{abstract}

\section{Introduction}

In this paper we analyze the compatibility of the principle of superposition for Maxwell fields and the principle of energy-momentum conservation. More exactly, we prove in a rigorous mathematical way, using the Clifford bundle formalism, that the energy and momentum of two distinct and otherwise arbitrary Maxwell fields (of finite energy) are additive. Let us now describe precisely our problem.

In a given inertial frame $\mathbf{I}=\partial / \partial x^{0} \in \sec T M$ in Minkowski spacetime (see Appendix for some details) with a natural adapted coordinates $\left\{x^{\mu}\right\}$ in Einstein- 
Lorentz-Poincaré gaug® 1 we have two identical antennas that are put on at time $t=-\tau$ and which are able to produce two distinct electromagnetic fields, which we take for simplicity, as being of the same time duration $\tau$. So, at time $t=0$ we have two electromagnetic field configurations denoted $F_{1}(0, \mathbf{x})$ and $F_{2}(0, \mathbf{x})$, which, of course, have compact support in a region $\mathfrak{R} \subset \mathbb{R}^{3}$ (the rest space of the inertial frame) and which we suppose are moving in opposite directions (to fix the ideas the $z$-direction). We suppose moreover that the two antennas are separated by a distance $D>\tau$, which means that one does not affect the other during the time they are generating the arbitrary electromagnetic field configurations $F_{1}(0, \mathbf{x})$ and $F_{2}(0, \mathbf{x})$. These fields can then be taken as Cauchy data for Maxwell equations and at any time $t$ satisfy Maxwell equation 2 in free space

$$
\partial F_{1}=0, \partial F_{2}=0
$$

Let $d$ be the distance between the wave fronts of the two pulses at $t=0$ measured along the $z$ axis. Now, at time $\mathfrak{t}=(d+\tau) / 2$ the pulses $F_{1}(\mathfrak{t}, \mathbf{x})$ and $F_{2}(\mathfrak{t}, \mathbf{x})$ (which will be always diffracted 3 in relation to the initial configurations $F_{1}(0, \mathbf{x})$ and $\left.F_{2}(0, \mathbf{x})\right)$ which move with the velocity of light $c=1$ fill the same region of space and generate a total electromagnetic field

$$
F(\mathfrak{t}, \mathbf{x})=F_{1}(\mathfrak{t}, \mathbf{x})+F_{2}(\mathfrak{t}, \mathbf{x}),
$$

which satisfy also the free Maxwell equation (with appropriate initial conditions) due to the principle of superposition valid for linear partial differential equations,

$$
\partial F=0 .
$$

In the Clifford bundle formalism the energy-momentum densities $\star \mathcal{T}^{\mathrm{a}} \in$ $\sec \bigwedge^{3} T^{*} M \hookrightarrow \sec \mathcal{C} \ell(M, \eta)(\mathbf{a}=0,1,2,3)$ of an electromagnetic field configuration $F$ is given by Riesz formulat (see Appendix)

$$
\star \mathcal{T}^{\mathbf{a}}=\frac{1}{2} \star\left(F \theta^{\mathbf{a}} \tilde{F}\right),
$$

and the energy-momentum $P_{F}^{\mathbf{a}}$ of the field configuration at time $t=\mathfrak{t}$ is given by

$$
P_{F}^{\mathbf{a}}=\int_{B_{2}} \star \mathcal{T}^{\mathbf{a}},
$$

\footnotetext{
${ }^{1}$ We use units where the velocity of light $c$ has the numerical value 1 and so the timelike coordinate $x^{0}=t$.

${ }^{2}$ No misprint here. Maxwell equation in free space $\boldsymbol{\partial} F=0$ is the equation of motion for an electromagnetic field configuration $F \in \sec \bigwedge^{2} T^{*} M \hookrightarrow \sec \mathcal{C} \ell(M, \eta)$, where $\mathcal{C} \ell(M, \eta)$ is the Clifford bundle of differential forms. See the Appendices for explanation of the symbols and the main definitions and [19] for a detailed exposition.

${ }^{3}$ This is a consequence of the non focusing theorem. See 26$]$ and also 3 .

${ }^{4}$ Notice that $\mathcal{T}_{\mathbf{a}}=\mathcal{T}_{\mathbf{a b}} \theta^{\mathbf{b}}$, where $\mathcal{T}_{\mathbf{a b}}$ are the components of the usual [15] energymomentum tensor of Maxwell theory. Discussions about the appropriateness for the use of the usual energy-momentum tensor for the description of energy-momentum propagation are given in 15, 17.
} 
wher 5 5 $B_{2}$ is contained in the constant time hypersurface $t=\mathfrak{t}$ in Minkowski spacetime.

Now, due to Eq.(2) we have

$$
\begin{aligned}
\star \mathcal{T}^{\mathbf{a}} & =\frac{1}{2} \star\left(F \theta_{\mathbf{a}} \tilde{F}\right)=\frac{1}{2} \star\left(\left(F_{1}+F_{2}\right) \theta^{\mathbf{a}}\left(\widetilde{F_{1}+F_{2}}\right)\right) \\
& =\frac{1}{2} \star\left(F_{1} \theta^{\mathbf{a}} \tilde{F}_{1}+F_{2} \theta^{\mathbf{a}} \tilde{F}_{2}+F_{1} \theta^{\mathbf{a}} \tilde{F}_{2}+F_{2} \theta^{\mathbf{a}} \tilde{F}_{1}\right) \\
& =\star \mathcal{T}_{1}^{\mathbf{a}}+\star \mathcal{T}_{2}^{\mathbf{a}}+\star \mathcal{K}^{\mathbf{a}}
\end{aligned}
$$

where

$$
\begin{aligned}
\star \mathcal{T}_{1}^{\mathbf{a}} & =\frac{1}{2} \star\left(F_{1} \theta^{\mathbf{a}} \tilde{F}_{1}\right), \star \mathcal{T}_{2}^{\mathbf{a}}=\frac{1}{2} \star\left(F_{2} \theta^{\mathbf{a}} \tilde{F}_{2}\right), \\
\star \mathcal{K}^{\mathbf{a}} & =\frac{1}{2} \star\left(F_{1} \theta^{\mathbf{a}} \tilde{F}_{2}+F_{2} \theta^{\mathbf{a}} \tilde{F}_{1}\right) .
\end{aligned}
$$

Then we have that

$$
P_{F}^{\mathrm{a}}=\int_{B_{2}} \star \mathcal{T}^{\mathrm{a}}=\int_{B_{2}} \star \mathcal{T}_{1}^{\mathrm{a}}+\int_{B_{2}} \star \mathcal{T}_{2}^{\mathrm{a}}+\int_{B_{2}} \star \mathcal{K}^{\mathrm{a}}
$$

We want to prove that the energy and momentum of the field configuration $F_{1}$ and $F_{2}$ at time $t=\mathfrak{t}$ (and indeed at any time) is additive, i.e., is given by

$$
P_{F}^{\mathbf{a}}=P_{F_{1}}^{\mathbf{a}}+P_{F_{2}}^{\mathbf{a}}, \mathbf{a}=0,1,2,3,
$$

with

$$
P_{F_{1}}^{\mathbf{a}}=\int_{B_{2}} \star \mathcal{T}_{1}^{\mathbf{a}}, P_{F_{2}}^{\mathbf{a}}=\int_{B_{2}} \star \mathcal{T}_{2}^{\mathrm{a}} .
$$

This problem is a nontrivial one, and has been not discussed in the literature in an appropriate and satisfactory way according to our view. For example, in [11] (written in 1980) the author said that he found the problem discussed in only two $([6,24)$ out of 50 textbooks he has examined. Moreover, from a few papers published in the literature, we found some good ideas, but none offers a rigorous solution for the problem. Worse, some papers and books [2, 9, 10] have very odd and/or dubious statements. Indeed, in [2] it is said that Eq.(8) implies in non conservation of energy-momentum. The statement about non conservation of energy-momentum is also done by the author of [9, 10] who says that results of recent experiments [7] endorse his statement6. In [8] the double slit interference with monochromatic waves 7 is analyzed and it is said that energy-momentum is conserved only after spatial average. On the other hand, e.g., (4] shows that Eq.(9) is the correct one for the case of two plane

\footnotetext{
${ }^{5}$ See Figure 1 for the defintion of regions $B_{1}, B_{2}, B_{3}, B_{3}^{\prime}, C_{1}, C_{2}, C_{1}^{\prime}$ and $C_{2}^{\prime}$.

${ }^{6} \mathrm{~A}$ more intelligible and realistic analysis of light transmission through two slits is given in 25 .

${ }^{7}$ Which, of course, do not have compact support in $\mathbb{R}^{3}$.
} 
waves moving in opposite directions, but since waves of this kind (which do not have compact support) have infinite energy when the integration in Eq.(8) is done in all space, his approach cannot in any way be considered satisfactory. In [1] it is proposed that energy-momentum tensors that differ from an exact differential must be considered equivalent. This is a good idea, if it could be proved (something which has not been done in [1]) that

$$
\star \mathcal{K}^{\mathbf{a}}=-d \star \mathcal{E}^{\mathbf{a}}
$$

for some $\star \mathcal{E}^{\mathbf{a}} \in \sec \bigwedge^{2} T^{*} M \hookrightarrow \sec \mathcal{C} \ell(M, \eta)$ which goes to zero at spatial infinity at time $t=\mathfrak{t}$, since in this case we can write using Stokes theorem that

$$
\int_{B_{2}} \star \mathcal{K}^{\mathbf{a}}=-\int_{B_{2}} d \star \mathcal{E}^{\mathbf{a}}=-\int_{\partial B_{2}} \star \mathcal{E}^{\mathbf{a}}=0
$$

In the Section 2 we show that this is indeed the case for our problem. In Section 3 we prove that the energies and momenta of two different superposed electromagnetic field configurations are indeed additive. In Section 4 we present our conclusions. The paper have 4 Appendices. Appendix A introduces the concept of Clifford bundles, some important Clifford algebra identities, the Hodge star operator as an algebraic operation, and the Dirac operator acting on sections of the Clifford bundle. Appendix B presents Maxwell equation $\partial F=J$ and the noticeable formula for the energy-momentum densities $\star \mathcal{T}^{\mathbf{a}}=\frac{1}{2} \star\left(F \theta^{\mathbf{a}} \widetilde{F}\right)$. In Appendix $\mathrm{C}$ we describe the main features of the standard cylinder in Minkowski spacetime need in the applications of the Stokes theorem in the main text and in Appendix D we recall for completeness the generalized Green's formula for differential forms.

\section{Proof that $\star \mathcal{K}^{\mathrm{a}}=-d \star \mathcal{E}^{\mathrm{a}}$}

From Maxwell theory it follows (see Appendix) that for any free electromagnetic field configuration that

$$
\left.\delta \mathcal{T}^{\mathrm{a}}=-\partial\right\lrcorner \mathcal{T}^{\mathrm{a}}=0 .
$$

Since we obviously have $\left.\boldsymbol{\partial}\lrcorner \mathcal{T}_{1}^{\mathrm{a}}=\boldsymbol{\partial}\right\lrcorner \mathcal{T}_{2}^{\mathrm{a}}=0$, we necessarily must have that $\partial\lrcorner \mathcal{K}^{\mathbf{a}}=0$, i.e.,

$$
\boldsymbol{\partial}\lrcorner \frac{1}{2}\left(F_{1} \theta^{\mathbf{a}} \tilde{F}_{2}+F_{2} \theta^{\mathbf{a}} \tilde{F}_{1}\right)=0 .
$$

To show that this is indeed the case, first, observe that

$$
\begin{aligned}
& F_{1} \theta^{\mathbf{a}} \tilde{F}_{2}=\left\langle F_{1} \theta^{\mathbf{a}} \tilde{F}_{2}\right\rangle_{1}+\left\langle F_{1} \theta^{\mathbf{a}} \tilde{F}_{2}\right\rangle_{3}, \\
& F_{2} \theta^{\mathbf{a}} \tilde{F}_{1}=\left\langle F_{2} \theta^{\mathbf{a}} \tilde{F}_{1}\right\rangle_{1}+\left\langle F_{2} \theta^{\mathbf{a}} \tilde{F}_{1}\right\rangle_{3} .
\end{aligned}
$$

When the previous equations are added, the terms $\left\langle F_{1} \theta^{\mathbf{a}} \tilde{F}_{2}\right\rangle_{3}$ and $\left\langle F_{2} \theta^{\mathbf{a}} \tilde{F}_{1}\right\rangle_{3}$ cancel and we have

$$
F_{1} \theta^{\mathbf{a}} \tilde{F}_{2}+F_{2} \theta^{\mathbf{a}} \tilde{F}_{1}=\left\langle F_{1} \theta^{\mathbf{a}} \tilde{F}_{2}+F_{2} \theta^{\mathbf{a}} \tilde{F}_{1}\right\rangle_{1} .
$$


Returning to Eq.(12) we see that we need only to calculate $\boldsymbol{\partial}\lrcorner\left\langle F_{1} \theta^{\mathbf{a}} \tilde{F}_{2}+F_{2} \theta^{\mathbf{a}} \tilde{F}_{1}\right\rangle_{1}$. We have,

$$
\begin{aligned}
& \boldsymbol{\partial}\lrcorner\left\langle F_{1} \theta^{\mathbf{a}} \tilde{F}_{2}+F_{2} \theta^{\mathbf{a}} \tilde{F}_{1}\right\rangle_{1} \\
& =\left\langle\boldsymbol{\partial}\left(F_{1} \theta^{\mathbf{a}} \tilde{F}_{2}\right)+\boldsymbol{\partial}\left(F_{2} \theta^{\mathbf{a}} \tilde{F}_{1}\right)\right\rangle_{0} \\
& =\left\langle\theta^{\mathbf{b}} D_{e_{\mathbf{b}}}\left(F_{1} \theta^{\mathbf{a}} \tilde{F}_{2}\right)+\theta^{\mathbf{b}} D_{e_{\mathbf{b}}}\left(F_{2} \theta^{\mathbf{a}} \tilde{F}_{1}\right)\right\rangle_{0}
\end{aligned}
$$

or

$$
\begin{aligned}
& \boldsymbol{\partial}\lrcorner\left\langle F_{1} \theta^{\mathbf{a}} \tilde{F}_{2}+F_{2} \theta^{\mathbf{a}} \tilde{F}_{1}\right\rangle_{1} \\
& =\left\langle\left(\theta^{\mathbf{b}} D_{e_{\mathbf{b}}} F_{1}\right) \theta^{\mathbf{a}} \tilde{F}_{2}+\theta^{\mathbf{b}} F_{1} D_{e_{\mathbf{b}}}\left(\theta^{\mathbf{a}}\right) \tilde{F}_{2}+\theta^{\mathbf{b}} F_{1} \theta^{\mathbf{a}} D_{e_{\mathbf{b}}} \tilde{F}_{2}\right. \\
& \left.+\left(\theta^{\mathbf{b}} D_{e_{\mathbf{b}}} F_{2}\right) \theta^{\mathbf{a}} \tilde{F}_{1}+\theta^{\mathbf{b}} F_{2} D_{e_{\mathbf{b}}}\left(\theta^{\mathbf{a}}\right) \tilde{F}_{1}+\theta^{\mathbf{b}} F_{2} \theta^{\mathbf{a}} D_{e_{\mathbf{b}}} \tilde{F}_{1}\right\rangle_{0} .
\end{aligned}
$$

On the other hand, from the Eq. (1) we have

$$
\theta^{\mathbf{b}} D_{e_{\mathbf{b}}} F_{1}=\boldsymbol{\partial} F_{1}=0 \quad \text { and } \quad \theta^{\mathbf{b}} D_{e_{\mathbf{b}}} F_{2}=\boldsymbol{\partial} F_{2}=0
$$

and recalling that $\theta^{\mathbf{a}}=\delta_{\mu}^{\mathbf{a}} d x^{\mu}$ and $e_{\mathbf{b}}=\delta_{\mathbf{b}}^{\mu} \partial / \partial x^{\mu}$, we have that $D_{e_{\mathbf{b}}} \theta^{\mathbf{a}}=0$. Then, Eq. (12) can be written as

$$
\boldsymbol{\partial}\lrcorner \frac{1}{2}\left\langle F_{1} \theta^{\mathbf{a}} \tilde{F}_{2}+F_{2} \theta^{\mathbf{a}} \tilde{F}_{1}\right\rangle_{1}=\frac{1}{2}\left\langle\theta^{\mathbf{b}} F_{1} \theta^{\mathbf{a}} D_{e_{\mathbf{b}}} \tilde{F}_{2}+\theta^{\mathbf{b}} F_{2} \theta^{\mathbf{a}} D_{e_{\mathbf{b}}} \tilde{F}_{1}\right\rangle_{0}
$$

Now we examine the term $\left\langle\theta^{\mathbf{b}} F_{1} \theta^{\mathbf{a}} D_{e_{\mathbf{b}}} \tilde{F}_{2}+\theta^{\mathbf{b}} F_{2} \theta^{\mathbf{a}} D_{e_{\mathbf{b}}} \tilde{F}_{1}\right\rangle_{0}$. First observe that

$$
\begin{aligned}
\theta^{\mathbf{b}}\left(F_{1} \theta^{\mathbf{a}} D_{e_{\mathbf{b}}} \tilde{F}_{2}\right) & =\theta^{\mathbf{b}}\left\langle F_{1} \theta^{\mathbf{a}} D_{e_{\mathbf{b}}} \tilde{F}_{2}\right\rangle_{1}+\theta^{\mathbf{b}}\left\langle F_{1} \theta^{\mathbf{a}} D_{e_{\mathbf{b}}} \tilde{F}_{2}\right\rangle_{3} \\
& \left.=\theta^{\mathbf{b}}\right\lrcorner\left\langle F_{1} \theta^{\mathbf{a}} D_{e_{\mathbf{b}}} \tilde{F}_{2}\right\rangle_{1}+\theta^{\mathbf{b}} \wedge\left\langle F_{1} \theta^{\mathbf{a}} D_{e_{\mathbf{b}}} \tilde{F}_{2}\right\rangle_{1} \\
& \left.+\theta^{\mathbf{b}}\right\lrcorner\left\langle F_{1} \theta^{\mathbf{a}} D_{e_{\mathbf{b}}} \tilde{F}_{2}\right\rangle_{3}+\theta^{\mathbf{b}} \wedge\left\langle F_{1} \theta^{\mathbf{a}} D_{e_{\mathbf{b}}} \tilde{F}_{2}\right\rangle_{3} .
\end{aligned}
$$

Then

$$
\begin{aligned}
\left\langle\theta^{\mathbf{b}} F_{1} \theta^{\mathbf{a}} D_{e_{\mathbf{b}}} \tilde{F}_{2}\right\rangle_{0} & \left.=\theta^{\mathbf{b}}\right\lrcorner\left\langle F_{1} \theta^{\mathbf{a}} D_{e_{\mathbf{b}}} \tilde{F}_{2}\right\rangle_{1} \\
& =\left\langle F_{1} \theta^{\mathbf{a}} D_{e_{\mathbf{b}}} \tilde{F}_{2}\right\rangle_{1}\left\llcorner\theta^{\mathbf{b}}\right. \\
& =\left\langle F_{1} \theta^{\mathbf{a}}\left(D_{e_{\mathbf{b}}} \tilde{F}_{2}\right) \theta^{\mathbf{b}}\right\rangle_{0} \\
& =\left\langle F_{1} \theta^{\mathbf{a}}\left(\tilde{F}_{2} \overleftarrow{\boldsymbol{\partial}}\right)\right\rangle_{0}
\end{aligned}
$$

where we use the symbol $\left(D_{e_{\mathbf{b}}} \tilde{F}_{2}\right) \theta^{\mathbf{b}}:=\tilde{F}_{2} \overleftarrow{\boldsymbol{\partial}}$. Since $\tilde{F}_{2} \overleftarrow{\boldsymbol{\partial}}=\widetilde{\left(\boldsymbol{\partial} F_{2}\right)}=0$, we have $\left\langle\theta^{\mathbf{b}} F_{1} \theta^{\mathbf{a}} D_{e_{\mathbf{b}}} \tilde{F}_{2}\right\rangle_{0}=0$. Analogously we get that $\left\langle\theta^{\mathbf{b}} F_{2} \theta^{\mathbf{a}} D_{e_{\mathbf{b}}} \tilde{F}_{1}\right\rangle_{0}=0$, and thus

$$
\left\langle\theta^{\mathbf{b}} F_{1} \theta^{\mathbf{a}} D_{e_{\mathbf{b}}} \tilde{F}_{2}+\theta^{\mathbf{b}} F_{2} \theta^{\mathbf{a}} D_{e_{\mathbf{b}}} \tilde{F}_{1}\right\rangle_{0}=0 .
$$


Now, using Eq.(21) in Eq. (18) we have

$$
\boldsymbol{\partial}\lrcorner \frac{1}{2}\left\langle F_{1} \theta^{\mathbf{a}} \tilde{F}_{2}+F_{2} \theta^{\mathbf{a}} \tilde{F}_{1}\right\rangle_{1}=0 .
$$

We just proved that indeed, $\delta \mathcal{K}^{\mathbf{a}}=0$, or what is the same, that

$$
d \star \mathcal{K}^{\mathbf{a}}=0,
$$

and since we are in Minkowski spacetime Poincaré's lemma implies that the 3 -form fields $\star \mathcal{K}^{\mathbf{a}} \in \sec \bigwedge^{3} T^{*} M \hookrightarrow \sec \mathcal{C} \ell(M, \eta)$ must be exact, i.e.,

$$
\star \mathcal{K}^{\mathbf{a}}=-d \star \mathcal{E}^{\mathbf{a}},
$$

or

$$
\delta \mathcal{E}^{\mathbf{a}}=-\mathcal{K}^{\mathbf{a}}
$$

\section{The Energies and Momenta of Two Different Superposed Electromagnetic Field Configura- tions are Additive.}

In this section the standard cylinder of Minkowski spacetime and its boundary submanifolds (see Figure) described in the Appendix will be used. We start our enterprise by recalling that since $\star \mathcal{T}^{\mathrm{a}}=\star \mathcal{T}_{1}^{\mathrm{a}}+\star \mathcal{T}_{2}^{\mathrm{a}}+\star \mathcal{K}^{\mathrm{a}}$ and $d \star \mathcal{T}^{\mathrm{a}}=$ $0, d \star \mathcal{T}_{1}^{\mathrm{a}}=0, d \star \mathcal{T}_{2}^{\mathrm{a}}=0$ and $d \star \mathcal{K}^{\mathbf{a}}=0$ we can use Stokes theorem to write

$$
\begin{aligned}
0 & =\int_{N} d \star \mathcal{K}^{\mathbf{a}}=-\int_{B_{1}^{\prime}} \star \mathcal{K}^{\mathbf{a}}+\int_{B_{2}^{\prime}} \star \mathcal{K}^{\mathbf{a}}+\int_{B_{3}^{\prime}} \star \mathcal{K}^{\mathbf{a}} \\
& =-\int_{B_{1}} \star \mathcal{K}^{\mathbf{a}}+\int_{B_{2}} \star \mathcal{K}^{\mathbf{a}}+\int_{B_{3}^{\prime}} \star \mathcal{K}^{\mathbf{a}}
\end{aligned}
$$

from where it follows that

$$
\int_{B_{1}} \star \mathcal{K}^{\mathbf{a}}=\int_{B_{2}} \star \mathcal{K}^{\mathbf{a}}+\int_{B_{3}^{\prime}} \star \mathcal{K}^{\mathbf{a}}
$$

Now, if we take into account Eq.(7) which defines $\star \mathcal{K}^{\mathbf{a}}$ we see immediately that $\int_{B_{3}^{\prime}} \star \mathcal{K}^{\mathbf{a}}=0$ because $\left.F_{1}\right|_{B_{3}^{\prime}}=0$ and $\left.F_{1}\right|_{B_{3}^{\prime}}=0$. Also, since (obviously) $\left.\star \mathcal{K}^{\mathbf{a}}\right|_{B_{1}}=0$ it follows that $\int_{B_{1}} \star \mathcal{K}^{\mathbf{a}}=0$ and we get that

$$
\int_{B_{2}} \star \mathcal{K}^{\mathbf{a}}=0
$$

even if $\left.\star \mathcal{K}^{\mathrm{a}}\right|_{B_{2}} \neq 0$ 
Using these results we can calculate the total energy of $F=F_{1}+F_{2}$ containing in $B_{2}$. We have, taking into account Eq.(6) and Eq.(28) that

$$
\begin{aligned}
\int_{B_{2}} \star \mathcal{T}^{\mathbf{a}} & =\int_{B_{2}} \star\left(\mathcal{T}_{1}^{\mathbf{a}}+\mathcal{T}_{2}^{\mathbf{a}}\right)+\int_{B_{2}} \star \mathcal{K}^{\mathbf{a}} \\
& =\int_{B_{2}} \star\left(\mathcal{T}_{1}^{\mathbf{a}}+\mathcal{T}_{2}^{\mathbf{a}}\right),
\end{aligned}
$$

i.e.,

$$
P_{F}^{\mathbf{a}}=P_{F_{1}}^{\mathbf{a}}+P_{F_{2}}^{\mathbf{a}},
$$

as we wanted to prove.

\section{Conclusions}

In this paper we proved that the energy and momenta of two free electromagnetic field configurations (which satisfy at any instant of time a free Maxwell equation and for each finite instant of time have compact support in $\mathbb{R}^{3}$ ) of finite energy are additive and thus there is no incompatibility between the principle of superposition of energy and the principle of energy-momentum conservations as suggested by some authors, quoted in Section 18 . We emphasize that our proof is made relatively simple due to the amazing power of the Clifford bundle formalism, and indeed we do not see how to do the calculations using the old Heaviside-Gibbs vector calculus, or even only the Cartan calculus of differential forms, since our proof depends deeply on the noticeable formula for the energy-momentum densities, namely $\star \mathcal{T}^{\mathbf{a}}=\frac{1}{2} F \theta^{\mathbf{a}} \tilde{F}$ which is valid for any electromagnetic field configuration $F$ satisfying Maxwell equation $\partial F=0$. We emphasize also that we have found there exist closed 2-forms $\star \mathcal{W}^{\mathrm{a}} \in \sec \bigwedge^{2} T^{*} M \hookrightarrow \sec \mathcal{C} \ell(M, \eta)$ satisfying generalized Maxwell equations $\partial \mathcal{W}^{\mathbf{a}}=\mathcal{T}^{\mathbf{a}}+\star M_{\mathbf{a}}$. This result that will be explored in future publications.

\section{A Clifford Bundles}

Let $\left(M, \eta, D, \tau_{\eta}, \uparrow\right)$ be Minkowski spacetime. $(M, \eta)$ is a four dimensional space oriented (by the volume form $\tau_{\eta}$ ) and time oriented (by the equivalence relation $\uparrow$, see [19]) Lorentzian manifold, with $M \simeq \mathbb{R}^{4}$ and $\eta \in \sec T_{2}^{0} M$ is a Lorentzian metric of signature $(1,3) . T^{*} M[T M]$ is the cotangent [tangent] bundle. $T^{*} M=\cup_{x \in M} T_{x}^{*} M, T M=\cup_{x \in M} T_{x} M$, and $T_{x} M \simeq T_{x}^{*} M \simeq \mathbb{R}^{1,3}$, where $\mathbb{R}^{1,3}$ is the Minkowski vector space . $D$ is the Levi-Civita connection of $\eta$, i.e., $D \eta=0, \mathbf{R}(D)=0$. Also $\mathbf{T}(D)=0, \mathbf{R}$ and $\mathbf{T}$ being respectively the torsion and curvature tensors. Let $\eta \in \sec T_{0}^{2} M$ be the metric on the cotangent bundle associated to $\eta \in \sec T_{2}^{0} M$. The Clifford bundle of differential forms $\mathcal{C}(M, \eta)$ is the bundle of algebras, i.e., $\mathcal{C}(M, \eta)=\cup_{x \in M} \mathcal{C}\left(T_{x}^{*} M\right)$,

\footnotetext{
${ }^{8}$ We observe that the same problem occur for all linear field theories, and indeed in reference ([11 [12, 21]) we have some discussion of the problem for sound (and other elastic) waves.
} 
where $\forall x \in M, \mathcal{C l}\left(T_{x}^{*} M\right)=\mathbb{R}_{1,3}$, the so called spacetime algebra. Recall also that $\mathcal{C}(M, \eta)$ is a vector bundle associated to the orthonormal frame bundle $\mathbf{P}_{\mathrm{SO}_{(1,3)}^{e}}(M)$, i.e., $\mathcal{C}(M, \eta)=P_{S O_{+(1,3)}}(M) \times_{a d} \mathbb{R}_{1,3}$ (see details in, e.g., [16, 13, 19]). For any $x \in M, \mathcal{C}\left(T_{x}^{*} M\right)$ is a linear space over the real field $\mathbb{R}$. Moreover, $\mathcal{C}\left(T_{x}^{*} M\right)$ is isomorphic to the Cartan algebra $\bigwedge T_{x}^{*} M$ of the cotangent space and $\bigwedge T_{x}^{*} M=\sum_{k=0}^{4} \bigwedge^{k} T_{x}^{*} M$, where $\bigwedge^{k} T_{x}^{*} M$ is the $\left(\begin{array}{l}4 \\ k\end{array}\right)$-dimensional space of $k$-forms. Then, sections of $\mathcal{C}(M, \eta)$ can be represented as a sum of non homogeneous differential forms. Let $\left\{x^{\mu}\right\}$ be coordinates in Einstein-LorentzPoincaré gauge for $M$ and let $\left\{e_{\mu}=\partial / \partial x^{\mu}\right\} \in \sec F M$ (the frame bundle) be an orthonormal basis for $T M$, i.e., $\eta\left(e_{\mu}, e_{\nu}\right)=\eta_{\mu \nu}=\operatorname{diag}(1,-1,-1,-1)$, Let $\gamma^{\nu}=d x^{\nu} \in \sec \bigwedge^{1} T^{*} M \hookrightarrow \sec \mathcal{C l}(M, \eta)(\nu=0,1,2,3)$ such that the set $\left\{\gamma^{\nu}\right\}$ is the dual basis of $\left\{e_{\mu}\right\}$, and of course, $\eta\left(\gamma^{\mu}, \gamma^{\nu}\right)=\eta^{\mu \nu}=\operatorname{diag}(1,-1,-1,-1)$. We introduce moreover the notations $\theta^{\mathbf{a}}=\delta_{\mu}^{\mathbf{a}} d x^{\mu}$ and $\mathbf{e}_{\mathbf{a}}=\delta_{\mathbf{a}}^{\mu} \frac{\partial}{\partial x^{\mu}}$. We say that $\left\{\mathbf{e}_{\mathbf{a}}\right\}$ is a section of the orthonormal frame bundle $\mathbf{P}_{\mathrm{SO}_{(1,3)}^{e}}(M)$ and its dual basis $\left\{\theta^{\mathbf{a}}\right\}$ is a section of the orthonormal coframe bundle (denoted $P_{\mathrm{SO}_{(1,3)}^{e}}^{e}(M)$ ).

\section{A.1 Clifford Product}

The fundamental Clifford product (in what follows to be denoted by juxtaposition of symbols) is generated by $\theta^{\mathbf{a}} \theta^{\mathbf{a}}+\theta^{\mathbf{b}} \theta^{\mathbf{a}}=2 \eta^{\mathbf{a b}}$ and if $\mathcal{C} \in \mathcal{C}(M, \eta)$ we have

$$
\mathcal{C}=s+v_{\mathbf{a}} \theta^{\mathbf{a}}+\frac{1}{2 !} b_{\mathbf{a b}} \theta^{\mathbf{a}} \theta^{\mathbf{b}}+\frac{1}{3 !} a_{\mathbf{a b c}} \theta^{\mathbf{a}} \theta^{\mathbf{b}} \theta^{\mathbf{c}}+p \theta^{5},
$$

where $\tau_{\eta}:=\theta^{5}=\theta^{0} \theta^{1} \theta^{2} \theta^{3}=d x^{0} d x^{1} d x^{2} d x^{3}$ is the volume element and $s, v_{\mathbf{a}}$, $b_{\mathbf{a b}}, a_{\mathbf{a b c}}, p \in \sec \bigwedge^{0} T^{*} M \hookrightarrow \sec \mathcal{C}(M, \eta)$.

Let $\mathcal{A}_{r}, \in \sec \bigwedge^{r} T^{*} M \hookrightarrow \sec \mathcal{C}(M, \eta), \mathcal{B}_{s} \in \sec \bigwedge^{s} T^{*} M \hookrightarrow \sec \mathcal{C}(M, \eta)$. For $r=s=1$, we define the scalar product as follows:

For $a, b \in \sec \bigwedge^{1} T^{*} M \hookrightarrow \sec \mathcal{C}(M, \eta)$,

$$
a \cdot b=\frac{1}{2}(a b+b a)=\eta(a, b) .
$$

We define also the exterior product $(\forall r, s=0,1,2,3)$ by

$$
\begin{aligned}
& \mathcal{A}_{r} \wedge \mathcal{B}_{s}=\left\langle\mathcal{A}_{r} \mathcal{B}_{s}\right\rangle_{r+s}, \\
& \mathcal{A}_{r} \wedge \mathcal{B}_{s}=(-1)^{r s} \mathcal{B}_{s} \wedge \mathcal{A}_{r},
\end{aligned}
$$

where \langle\rangle$_{k}$ is the component in $\Lambda^{k} T^{*} M$ (projection) of the Clifford field. The exterior product is extended by linearity to all sections of $\mathcal{C}(M, \eta)$..

For $\mathcal{A}_{r}=a_{1} \wedge \ldots \wedge a_{r}, \mathcal{B}_{r}=b_{1} \wedge \ldots \wedge b_{r}$, the scalar product is defined here as follows,

$$
\begin{aligned}
\mathcal{A}_{r} \cdot \mathcal{B}_{r} & =\left(a_{1} \wedge \ldots \wedge a_{r}\right) \cdot\left(b_{1} \wedge \ldots \wedge b_{r}\right) \\
& =\left|\begin{array}{ccc}
a_{1} \cdot b_{1} & \ldots . & a_{1} \cdot b_{r} \\
\ldots \ldots \ldots . & \ldots . & \ldots \ldots \ldots \ldots \\
a_{r} \cdot b_{1} & \ldots . & a_{r} \cdot b_{r}
\end{array}\right| .
\end{aligned}
$$


We agree that if $r=s=0$, the scalar product is simple the ordinary product in the real field.

Also, if $r \neq s$, then $\mathcal{A}_{r} \cdot \mathcal{B}_{s}=0$. Finally, the scalar product is extended by linearity for all sections of $\mathcal{C}(M, \eta)$. by

For $r \leq s, \mathcal{A}_{r}=a_{1} \wedge \ldots \wedge a_{r}, \mathcal{B}_{s}=b_{1} \wedge \ldots \wedge b_{s}$ we define the left contraction

$$
\lrcorner:\left(\mathcal{A}_{r}, \mathcal{B}_{s}\right) \mapsto \mathcal{A}_{r}\right\lrcorner \mathcal{B}_{s}=\sum_{i_{1}<\ldots<i_{r}} \epsilon^{i_{1} \ldots i_{s}}\left(a_{1} \wedge \ldots \wedge a_{r}\right) \cdot\left(b_{i_{1}} \wedge \ldots \wedge b_{i_{r}}\right)^{\sim} b_{i_{r}+1} \wedge \ldots \wedge b_{i_{s}}
$$

where $\sim$ is the reverse mapping (reversion) defined by

$$
\sim: \sec \bigwedge^{p} T^{*} M \ni a_{1} \wedge \ldots \wedge a_{p} \mapsto a_{p} \wedge \ldots \wedge a_{1}
$$

and extended by linearity to all sections of $\mathcal{C}(M, \eta)$. We agree that for $\alpha, \beta \in$ $\sec \bigwedge^{0} T^{*} M$ the contraction is the ordinary (pointwise) product in the real field and that if $\alpha \in \sec \bigwedge^{0} T^{*} M \hookrightarrow \mathcal{C}(M, \eta), \mathcal{A}_{r}, \in \sec \bigwedge^{r} T^{*} M \hookrightarrow \mathcal{C}(M, \eta)$, $\mathcal{B}_{s} \in \sec \bigwedge^{s} T^{*} M \hookrightarrow \mathcal{C}(M, \eta)$ then $\left.\left.\left(\alpha \mathcal{A}_{r}\right)\right\lrcorner \mathcal{B}_{s}=\mathcal{A}_{r}\right\lrcorner\left(\alpha \mathcal{B}_{s}\right)$. Left contraction is extended by linearity to all pairs of elements of sections of $\mathcal{C}(M, \eta)$, i.e., for $\mathcal{A}, \mathcal{B} \in \sec \mathcal{C}(M, \eta)$

$$
\left.\mathcal{A}\lrcorner \mathcal{B}=\sum_{r, s}\langle\mathcal{A}\rangle_{r}\right\lrcorner\langle\mathcal{B}\rangle_{s}, r \leq s .
$$

It is also necessary to introduce the operator of right contraction denoted by $\llcorner$. The definition is obtained from the one presenting the left contraction with the imposition that $r \geq s$ and taking into account that now if $\mathcal{A}_{r} \in \sec \bigwedge^{r} T^{*} M \hookrightarrow \mathcal{C}(M, \eta), \mathcal{B}_{s} \in \sec \bigwedge^{s} T^{*} M \hookrightarrow \mathcal{C}(M, \eta)$ then $\mathcal{A}_{r}\left\llcorner\left(\alpha \mathcal{B}_{s}\right)=\right.$ $\left(\alpha \mathcal{A}_{r}\right)\left\llcorner\mathcal{B}_{s}\right.$.

The main formulas used in the present paper can be obtained (details may be found in [19]) from the following ones (where $a \in \sec \bigwedge^{1} T^{*} M \hookrightarrow \sec \mathcal{C}(M, \eta)$ ):

$$
\begin{aligned}
a \mathcal{B}_{s} & =a\lrcorner \mathcal{B}_{s}+a \wedge \mathcal{B}_{s}, \mathcal{B}_{s} a=\mathcal{B}_{s}\left\llcorner a+\mathcal{B}_{s} \wedge a,\right. \\
a\lrcorner \mathcal{B}_{s} & =\frac{1}{2}\left(a \mathcal{B}_{s}-(-1)^{s} \mathcal{B}_{s} a\right), \\
\left.\mathcal{A}_{r}\right\lrcorner \mathcal{B}_{s} & =(-1)^{r(s-r)} \mathcal{B}_{s}\left\llcorner\mathcal{A}_{r},\right. \\
a \wedge \mathcal{B}_{s}= & \frac{1}{2}\left(a \mathcal{B}_{s}+(-1)^{s} \mathcal{B}_{s} a\right), \\
\mathcal{A}_{r} \mathcal{B}_{s}= & \left\langle\mathcal{A}_{r} \mathcal{B}_{s}\right\rangle_{|r-s|}+\left\langle\mathcal{A}_{r} \mathcal{B}_{s}\right\rangle_{|r-s|+2}+\ldots+\left\langle\mathcal{A}_{r} \mathcal{B}_{s}\right\rangle_{|r+s|} \\
& =\sum_{k=0}^{m}\left\langle\mathcal{A}_{r} \mathcal{B}_{s}\right\rangle_{|r-s|+2 k} \\
\mathcal{A}_{r} \cdot \mathcal{B}_{r}= & \left.\mathcal{B}_{r} \cdot \mathcal{A}_{r}=\widetilde{\mathcal{A}}_{r}\right\lrcorner \mathcal{B}_{r}=\mathcal{A}_{r}\left\llcorner\widetilde{\mathcal{B}}_{r}=\left\langle\widetilde{\mathcal{A}}_{r} \mathcal{B}_{r}\right\rangle_{0}=\left\langle\mathcal{A}_{r} \widetilde{\mathcal{B}}_{r}\right\rangle_{0},\right.
\end{aligned}
$$




$$
\begin{aligned}
\langle\mathcal{A B}\rangle_{r} & =(-1)^{r(r-1) / 2}\langle\widetilde{\mathcal{B}} \widetilde{\mathcal{A}}\rangle_{r} \\
\left\langle\mathcal{A}_{r} \mathcal{B}_{s}\right\rangle_{r} & =\left\langle\widetilde{\mathcal{B}}_{s} A_{r}\right\rangle_{r}=(-1)^{s(s-1) / 2}\left\langle\mathcal{B}_{s} \mathcal{A}_{r}\right\rangle_{r}, \\
\left\langle\mathcal{A}_{r} \mathcal{B}_{s} \mathcal{C}_{t}\right\rangle_{q} & =(-1)^{\varepsilon}\left\langle\mathcal{C}_{t} \mathcal{B}_{s} \mathcal{A}_{r}\right\rangle_{q}, \\
\varepsilon & =\frac{1}{2}\left(q^{2}+r^{2}+s^{2}+t^{2}-q-r-s-t\right)
\end{aligned}
$$

\section{A.2 Hodge Star Operator}

Let $\star$ be the Hodge star operator, i.e., the mapping

$$
\star: \bigwedge^{k} T^{*} M \rightarrow \bigwedge^{4-k} T^{*} M, \mathcal{A}_{k} \mapsto \star \mathcal{A}_{k}
$$

where for $\mathcal{A}_{k} \in \sec \bigwedge^{k} T^{*} M \hookrightarrow \mathcal{C l}(M, \eta)$

$$
\left[\mathcal{B}_{k} \cdot \mathcal{A}_{k}\right] \tau_{\eta}=\mathcal{B}_{k} \wedge \star \mathcal{A}_{k}, \forall \mathcal{B}_{k} \in \sec \bigwedge^{k} T^{*} M \hookrightarrow \sec \mathcal{C}(M, \eta) .
$$

$\tau_{\eta}=\theta^{5} \in \bigwedge^{4} T^{*} M$ is a standard volume element. Then we can verify that

$$
\star \mathcal{A}_{k}=\widetilde{\mathcal{A}}_{k} \tau_{\eta}=\widetilde{\mathcal{A}}_{k} \theta^{5} .
$$

\section{A.3 Dirac Operator}

Let $d$ and $\delta$ be respectively the differential and Hodge codifferential operators acting on sections of $\sec \bigwedge^{k} T^{*} M \hookrightarrow \sec \mathcal{C}(M, \eta)$. If $\mathcal{A}_{p} \in \sec \bigwedge^{p} T^{*} M \hookrightarrow$ $\sec \mathcal{C}(M, \eta)$, then $\delta \mathcal{A}_{p}=(-1)^{p} \star^{-1} d \star \mathcal{A}_{p}$, with $\star^{-1} \star=$ identity.

The Dirac operator acting on sections of $\mathcal{C}(M, g)$ is the invariant first order differential operator

$$
\boldsymbol{\partial}=\theta^{\mathrm{a}} D_{\mathbf{e}_{\mathbf{a}}}
$$

\section{A.3.1 $\quad D_{\mathrm{e}_{\mathrm{a}}} \mathcal{A}$}

The reciprocal basis of $\left\{\theta^{\mathbf{b}}\right\}$ is denoted $\left\{\theta_{\mathbf{a}}\right\}$ and we have $\theta_{\mathbf{a}} \cdot \theta_{\mathbf{b}}=\eta_{\mathbf{a b}}\left(\eta_{\mathbf{a b}}=\right.$ $\operatorname{diag}(1,-1,-1,-1))$. Also,

$$
D_{\mathbf{e}_{\mathbf{a}}} \theta^{\mathbf{b}}=-\omega_{\mathbf{a c}}^{\mathbf{b}} \theta^{\mathbf{c}}=-\omega_{\mathbf{a}}^{\mathbf{b c}} \theta_{\mathbf{c}}
$$

with $\omega_{\mathbf{a}}^{\mathbf{b c}}=-\omega_{\mathbf{a}}^{\mathbf{c b}}$, and $\omega_{\mathbf{a}}^{\mathbf{b c}}=\eta^{\mathbf{b k}} \omega_{\mathbf{k a l}} \eta^{\mathbf{c l}}, \omega_{\mathbf{a b c}}=\eta_{\mathbf{a d}} \omega_{\mathbf{b c}}^{\mathbf{d}}=-\omega_{\mathbf{c b a}}$. Defining

$$
\omega_{\mathbf{a}}=\frac{1}{2} \omega_{\mathbf{a}}^{\mathbf{b c}} \theta_{\mathbf{b}} \wedge \theta_{\mathbf{c}} \in \sec \bigwedge^{2} T^{*} M \hookrightarrow \sec \mathcal{C}(M, \eta),
$$

we have (by linearity) that for any $\mathcal{A} \in \sec \wedge T^{*} M \hookrightarrow \sec \mathcal{C}(M, \eta)$

$$
D_{\mathbf{e}_{\mathbf{a}}} \mathcal{A}=\partial_{\mathbf{e}_{\mathbf{a}}} \mathcal{A}+\frac{1}{2}\left[\omega_{\mathbf{a}}, \mathcal{A}\right],
$$

where $\partial_{\mathbf{e}_{\mathbf{a}}}$ is the Pfaff derivative9

\footnotetext{
${ }^{9}$ E.g., if $A=\frac{1}{p !} A_{\mathbf{i}_{1} \ldots \mathbf{i}_{p}} \theta^{\mathbf{i}_{1}} \ldots \theta^{\cdot \mathbf{i}_{p}}$ then $\partial_{\mathbf{e}_{\mathbf{a}}} A=\frac{1}{p !}\left[\mathbf{e}_{\mathbf{a}}\left(A_{\mathbf{i}_{1} \ldots \mathbf{i}_{p}}\right)\right] \theta^{\mathbf{i}_{1}} \ldots \theta^{\cdot \mathbf{i}_{p}}$.
} 
A.3.2 $\partial=d-\delta$

Using Eq.(44) we can easily show the very important result:

$$
\begin{aligned}
\partial \mathcal{A} & =\partial \wedge \mathcal{A}+\partial\lrcorner \mathcal{A}=d \mathcal{A}-\delta \mathcal{A}, \\
\partial \wedge \mathcal{A} & =d \mathcal{A}, \quad \partial\lrcorner \mathcal{A}=-\delta \mathcal{A} .
\end{aligned}
$$

\section{B Maxwell Equation}

Eq.(45) permit us to write the Maxwell equations

$$
d F=0, \delta F=-J
$$

for $F \in \sec \bigwedge^{2} T^{*} M \hookrightarrow \sec \mathcal{C}(M, \eta)$ as a single equation (Maxwell equation),

$$
\partial F=J .
$$

\section{B.1 The Noticeable Riesz Formula $\mathcal{T}_{\mathbf{a}}=\frac{1}{2} F \theta_{\mathbf{a}} \tilde{F}$}

We now prove that the energy-momentum densities $\star \mathcal{T}_{\mathbf{a}}$ of the Maxwell field can be written in the Clifford bundle formalism as:

$$
\star \mathcal{T}_{\mathbf{a}}=\frac{1}{2} \star\left(F \theta_{\mathbf{a}} \tilde{F}\right) \in \sec \bigwedge^{3} T^{*} M \hookrightarrow \sec \mathcal{C} \ell(M, \eta) .
$$

To derive Eq.(48) we start from the Maxwell Lagrangian

$$
\mathcal{L}_{m}=\frac{1}{2} F \wedge \star F
$$

where $F=\frac{1}{2} F_{\mathbf{a b}} \theta^{\mathbf{a}} \wedge \theta^{\mathbf{b}}:=\frac{1}{2} F_{\mathbf{a b}} \theta^{\mathbf{a b}} \in \sec \bigwedge^{2} T M \hookrightarrow \sec \mathcal{C} \ell(M, \eta)$ is the electromagnetic field. Now, denoting by $\boldsymbol{\delta}$ the variational symbo 11 we can easily verify that

$$
\left.\boldsymbol{\delta} \star \theta^{\mathbf{a b}}=\boldsymbol{\delta} \theta^{\mathbf{c}} \wedge\left[\theta_{\mathbf{c}}\right\lrcorner \star \theta^{\mathbf{a b}}\right] .
$$

Moreover, in general $\boldsymbol{\delta}$ and $\star$ do not commute. Indeed, for any $\mathcal{A}_{p} \in \sec \bigwedge^{p} T^{*} M \hookrightarrow$ $\sec \mathcal{C} \ell(M, \eta)$ we have

$$
\begin{aligned}
{[\boldsymbol{\delta}, \star] \mathcal{A}_{p} } & =\boldsymbol{\delta} \star \mathcal{A}_{p}-\star \delta \mathcal{A}_{p} \\
& \left.\left.=\boldsymbol{\delta} \theta^{\mathbf{a}} \wedge\left(\theta_{\mathbf{a}}\right\lrcorner \star \mathcal{A}_{p}\right)-\star\left[\boldsymbol{\delta} \theta^{\mathbf{a}} \wedge\left(\theta_{\mathbf{a}}\right\lrcorner \mathcal{A}_{p}\right)\right] .
\end{aligned}
$$

\footnotetext{
${ }^{10}$ The formula $\mathcal{T}_{\mathbf{a}}=\frac{1}{2} F \theta_{\mathbf{a}} \tilde{F}$ has been first obtained (but, not using the algebraic derivatives of the Lagrangian density) by M. Riesz in 1947 [18 and it has been rediscovered by Hestenes in 1996 [5] (which also does not use the algebraic derivatives of the Lagrangian density). Algebraic derivatives of homogenous form fields has been described, e.g., in Thirring's book 23.

${ }^{11}$ Please, do not confuse the variational symbol $\boldsymbol{\delta}$ with the symbol $\delta$ of the Hodge coderiviative.
} 
Multiplying both members of Eq.(50) with $\mathcal{A}_{p}=F$ on the right by $F \wedge$ we get

$$
\left.\left.F \wedge \boldsymbol{\delta} \star F=F \wedge \star \delta F+F \wedge\left\{\boldsymbol{\delta} \theta^{\mathbf{a}} \wedge\left(\theta_{\mathbf{a}}\right\lrcorner \star F\right)-\star\left[\boldsymbol{\delta} \theta^{\mathbf{a}} \wedge\left(\theta_{\mathbf{a}}\right\lrcorner F\right)\right]\right\} .
$$

Next we sum $\boldsymbol{\delta} F \wedge \star F$ to both members of the above equation obtaining

$$
\left.\left.\boldsymbol{\delta}(F \wedge \star F)=2 \boldsymbol{\delta} F \wedge \star F+\boldsymbol{\delta} \theta^{\mathbf{a}} \wedge\left[F \wedge\left(\theta_{\mathbf{a}}\right\lrcorner \star F\right)-\left(\theta_{\mathbf{a}}\right\lrcorner F\right) \wedge \star F\right] .
$$

Then, it follows (see, [19, 20, for details) that i 12 $\delta \theta^{\mathbf{a}}=-£_{\xi} \theta^{\mathrm{a}}$, for some diffeomorphism generated by the vector field $\xi$ that

$$
\left.\left.\star \mathcal{T}_{\mathbf{a}}=\frac{\partial \mathcal{L}_{m}}{\partial \theta^{\mathbf{a}}}=\frac{1}{2}\left[F \wedge\left(\theta_{\mathbf{a}}\right\lrcorner \star F\right)-\left(\theta_{\mathbf{a}}\right\lrcorner F\right) \wedge \star F\right] .
$$

Now,

$$
\left.\left.\left.\left.\left.\left(\theta_{\mathbf{a}}\right\lrcorner F\right) \wedge \star F=-\star\left[\left(\theta_{\mathbf{a}}\right\lrcorner F\right)\right\lrcorner F\right]=-\left[\left(\theta_{\mathbf{a}}\right\lrcorner F\right)\right\lrcorner F\right] \tau_{\eta}
$$

and using also the identity [19]

$$
\left.\left.\left(\theta_{\mathbf{a}}\right\lrcorner F\right) \wedge \star F=\theta_{\mathbf{a}}(F \cdot F) \tau_{\eta}-F \wedge\left(\theta_{\mathbf{a}}\right\lrcorner \star F\right) .
$$

we get

$$
\begin{aligned}
\left.\left.\frac{1}{2}\left[F \wedge\left(\theta_{\mathbf{a}}\right\lrcorner \star F\right)-\left(\theta_{\mathbf{a}}\right\lrcorner F\right) \wedge \star F\right] & \left.\left.=\frac{1}{2}\left\{\theta_{\mathbf{a}}(F \cdot F) \tau_{\eta}-\left(\theta_{\mathbf{a}}\right\lrcorner F\right) \wedge \star F-\left(\theta_{\mathbf{a}}\right\lrcorner F\right) \wedge \star F\right\} \\
& \left.=\frac{1}{2}\left\{\theta_{\mathbf{a}}(F \cdot F) \tau_{\eta}-2\left(\theta_{\mathbf{a}}\right\lrcorner F\right) \wedge \star F\right\} \\
& \left.\left.=\frac{1}{2}\left\{\theta_{\mathbf{a}}(F \cdot F) \tau_{\eta}+2\left[\left(\theta_{\mathbf{a}}\right\lrcorner F\right)\right\lrcorner F\right] \tau_{\eta}\right\} \\
& \left.\left.=\star\left(\frac{1}{2} \theta_{\mathbf{a}}(F \cdot F)+\left(\theta_{\mathbf{a}}\right\lrcorner F\right)\right\lrcorner F\right)=\frac{1}{2} \star\left(F \theta_{\mathbf{a}} \tilde{F}\right),
\end{aligned}
$$

where in writing the last line we used the identity

$$
\left.\left.\frac{1}{2} F n \tilde{F}=(n\lrcorner F\right)\right\lrcorner F+\frac{1}{2} n(F \cdot F),
$$

whose proof is as follows:

$$
\begin{aligned}
(n\lrcorner F)\lrcorner F+\frac{1}{2} n(F \cdot F) & \left.\left.=\frac{1}{2}[(n\lrcorner F) F-F(n\lrcorner F\right)\right]+\frac{1}{2} n(F \cdot F) \\
& =\frac{1}{4}[n F F-F n F-F n F+F F n]+\frac{1}{2} n(F \cdot F) \\
& =-\frac{1}{2} F n F+\frac{1}{4}[-2 n(F \cdot F)+n(F \wedge F)+(F \wedge F) n]+\frac{1}{2} n(F \cdot F) \\
& =-\frac{1}{2} F n F+-\frac{1}{2} n(F \cdot F)+\frac{1}{2} n \wedge(F \wedge F)+\frac{1}{2} n(F \cdot F) \\
& =-\frac{1}{2} F n F=\frac{1}{2} F n \tilde{F} .
\end{aligned}
$$

\footnotetext{
$12 £_{\xi}$ denotes the Lie derivative in the direction of the vector field $\xi$.
} 
valid for any $n \in \sec \bigwedge^{1} T^{*} M \hookrightarrow \sec \mathcal{C} \ell(M, \eta)$ and $F \in \sec \bigwedge^{2} T^{*} M \hookrightarrow$ $\sec \mathcal{C} \ell(M, \eta)$.

For completeness and presentation of some more tricks of the trade we detail the proof that $\mathcal{T}_{\mathbf{a}} \cdot \theta_{\mathbf{b}}=\mathcal{T}_{\mathbf{b}} \cdot \theta_{\mathbf{a}}$.

$$
\begin{aligned}
\mathcal{T}_{\mathbf{a}} \cdot \theta_{\mathbf{b}} & =-\frac{1}{2}\left\langle F \theta_{\mathbf{a}} F \theta_{\mathbf{b}}\right\rangle_{0}=-\left\langle\left(F\left\llcorner\theta_{\mathbf{a}}\right) F \theta_{\mathbf{b}}\right\rangle_{0}-\frac{1}{2}\left\langle\left(\theta_{\mathbf{a}}\right\lrcorner F+\theta_{\mathbf{a}} \wedge F\right) F \theta_{\mathbf{b}}\right\rangle_{0} \\
& =-\left\langle\left(F\left\llcorner\theta_{\mathbf{a}}\right) F \theta_{\mathbf{b}}\right\rangle_{0}-\frac{1}{2}\left\langle\left(\theta_{\mathbf{a}} F F \theta_{\mathbf{b}}\right\rangle_{0}\right.\right. \\
& =-\left\langle\left(F \llcorner \theta _ { \mathbf { a } } ) \left( F\left\llcorner\theta_{\mathbf{b}}\right)+\left(F\left\llcorner\theta_{\mathbf{a}}\right)\left(F \wedge \theta_{\mathbf{b}}\right)\right\rangle_{0}+\frac{1}{2}\left\langle\theta_{\mathbf{a}}(F \cdot F) \theta^{\mathbf{b}}\right\rangle_{0}-\frac{1}{2}\left\langle\theta_{\mathbf{a}}(F \wedge F) \theta_{\mathbf{b}}\right\rangle_{0}\right.\right.\right. \\
& =-\left\langle\left( F\left\llcorner\theta_{\mathbf{a}}\right)\left(F\left\llcorner\theta_{\mathbf{b}}\right)\right\rangle_{0}+\frac{1}{2}\left\langle(F \cdot F)\left(\theta_{\mathbf{a}} \cdot \theta_{\mathbf{b}}\right)\right\rangle_{0}\right.\right. \\
& =-\left(F \llcorner \theta _ { \mathbf { b } } ) \cdot \left(F\left\llcorner\theta_{\mathbf{a}}\right)+\frac{1}{2}(F \cdot F)\left(\theta_{\mathbf{b}} \cdot \theta_{\mathbf{a}}\right)=\mathcal{T}_{\mathbf{b}} \cdot \theta_{\mathbf{a}} .\right.\right.
\end{aligned}
$$

Note moreover that

$$
\mathcal{T}_{\mathbf{a b}}=\mathcal{T}_{\mathbf{a}} \cdot \theta_{\mathbf{b}}=-\eta^{\mathbf{c l}} F_{\mathbf{a c}} F_{\mathbf{b l}}+\frac{1}{4} F_{\mathbf{c d}} F^{\mathbf{c d}} \eta_{\mathbf{a b}},
$$

a well known result.

Of course, for the free electromagnetic field we have that $d \star \mathcal{T}^{\mathbf{a}}=0$, which is equivalent to $\left.\delta \mathcal{T}^{\mathbf{a}}=-\boldsymbol{\partial}\right\lrcorner \mathcal{T}^{\mathbf{a}}=0$. Indeed, observe that

$$
\begin{aligned}
\left.\boldsymbol{\partial}\lrcorner \mathcal{T}^{\mathbf{a}}=\boldsymbol{\partial}\right\lrcorner & \frac{1}{2}\left(F \theta^{\mathbf{a}} \tilde{F}\right) \\
& =\frac{1}{2}\left\langle\boldsymbol{\partial}\left(F \theta^{\mathbf{a}} \tilde{F}\right)\right\rangle_{0} \\
& =\frac{1}{2}\left\langle(\boldsymbol{\partial} F) \theta^{\mathbf{a}} \tilde{F}+\theta^{\mathbf{b}}\left(F \theta^{\mathbf{a}} D_{e_{\mathbf{b}}} \tilde{F}\right)\right\rangle_{0} \\
& =\frac{1}{2}\left\langle\theta^{\mathbf{b}}\left(F \theta^{\mathbf{a}} D_{e_{\mathbf{b}}} \tilde{F}\right)\right\rangle_{0},
\end{aligned}
$$

where we used that $\boldsymbol{\partial} F=0$. Now,

$$
\begin{aligned}
\theta^{\mathbf{b}}\left(F \theta^{\mathbf{a}} D_{e_{\mathbf{b}}} \tilde{F}\right) & =\theta^{\mathbf{b}}\left\langle F \theta^{\mathbf{a}} D_{e_{\mathbf{b}}} \tilde{F}\right\rangle_{1}+\theta^{\mathbf{b}}\left\langle F \theta^{\mathbf{a}} D_{e_{\mathbf{b}}} \tilde{F}\right\rangle_{3} \\
& \left.=\theta^{\mathbf{b}}\right\lrcorner\left\langle F \theta^{\mathbf{a}} D_{e_{\mathbf{b}}} \tilde{F}\right\rangle_{1}+\theta^{\mathbf{b}} \wedge\left\langle F \theta^{\mathbf{a}} D_{e_{\mathbf{b}}} \tilde{F}\right\rangle_{1} \\
& \left.+\theta^{\mathbf{b}}\right\lrcorner\left\langle F \theta^{\mathbf{a}} D_{e_{\mathbf{b}}} \tilde{F}\right\rangle_{3}+\theta^{\mathbf{b}} \wedge\left\langle F \theta^{\mathbf{a}} D_{e_{\mathbf{b}}} \tilde{F}\right\rangle_{3} .
\end{aligned}
$$

Then

$$
\begin{aligned}
\left\langle\theta^{\mathbf{b}}\left(F \theta^{\mathbf{a}} D_{e_{\mathbf{b}}} \tilde{F}\right)\right\rangle_{0} & \left.=\theta^{\mathbf{b}}\right\lrcorner\left\langle F \theta^{\mathbf{a}} D_{e_{\mathbf{b}}} \tilde{F}\right\rangle_{1}=\left\langle F \theta^{\mathbf{a}} D_{e_{\mathbf{b}}} \tilde{F}\right\rangle_{1}\left\llcorner\theta^{\mathbf{b}}\right. \\
& =\left\langle F \theta^{\mathbf{a}} D_{e_{\mathbf{b}}} \tilde{F} \theta^{\mathbf{b}}\right\rangle_{0} \\
& =\left\langle F \theta^{\mathbf{a}} \widetilde{(\boldsymbol{\partial} F)}\right\rangle_{0}=0,
\end{aligned}
$$

where we used the symbol $\widetilde{(\boldsymbol{\partial F})}:=D_{e_{\mathbf{b}}} \tilde{F} \theta^{\mathbf{b}}$ and the fact that $\widetilde{(\boldsymbol{\partial} F)}=0$. 


\section{B.2 Enter New Maxwell Like Equations $d \star \mathcal{W}^{\mathrm{a}}=-\star \mathcal{T}^{\mathrm{a}}$, $d \mathcal{W}^{\mathrm{a}}=\star M^{\mathrm{a}}$}

Let $\star \mathcal{T}^{\mathbf{a}}=\frac{1}{2} \star\left(F \theta^{\mathbf{a}} \tilde{F}\right) \in \sec \bigwedge^{3} T^{*} M \hookrightarrow \sec \mathcal{C} \ell(M, \eta)$ be the energy-momentum densities of a free electromagnetic field configuration $F \in \sec \bigwedge^{2} T^{*} M \hookrightarrow \sec \mathcal{C} \ell(M, \eta)$ $(\boldsymbol{\partial} F=0)$. As we already know, we have

$$
\left.-\delta \mathcal{T}^{\mathrm{a}}=\partial\right\lrcorner \mathcal{T}^{\mathrm{a}}=0 .
$$

Eq. (55) is equivalent to $d \star \mathcal{T}^{\mathbf{a}}=0$ and since we are in Minkowski spacetime there must exist $\mathcal{W}^{\mathbf{a}} \in \sec \bigwedge^{2} T^{*} M \hookrightarrow \sec \mathcal{C} \ell(M, \eta)$ such that

$$
-\mathcal{T}^{\mathrm{a}}=\delta \mathcal{W}^{\mathrm{a}}
$$

Of course, we must also have

$$
d \mathcal{W}^{\mathbf{a}}=\star M^{\mathbf{a}}
$$

for some $M^{\mathrm{a}} \in \sec \bigwedge^{1} T^{*} M \hookrightarrow \sec \mathcal{C} \ell(M, \eta)$. Eqs. (56) and (57) may be writen as $\partial \mathcal{W}^{\mathbf{a}}=\star M^{\mathbf{a}}+\mathcal{T}^{\mathbf{a}}$. In another publication [14 we determine the explicit form of the $\mathcal{W}^{\mathbf{a}}$ and the $M^{\mathbf{a}}$.

\section{Standard Cylinder $N$ in Minkowski Space- time and its Boundary Submanifolds}

Let $N$ be the standard cylinder (Figure 1 at the end of the paper) [22] in Minkowski spacetime described in the Einstein-Lorentz-Poincaré coordinates $\left\{x^{\mu}\right\}$ naturally adapted to a inertial frame $\mathbf{I}=\partial / \partial x^{0}$ by

$$
\left.N=\left\{\left(x^{0}, x^{1}, x^{2}, x^{3}\right)\right\} \mid \sum_{i=1}^{3} x^{i} x^{i} \leq r^{\prime}, 0 \leq x^{0} \leq \mathfrak{t}\right\}
$$

The boundary manifolds of $N$ are the following submanifolds of $M$,

$$
\begin{aligned}
& \left.B_{1}^{\prime}=\left\{\left(0, x^{1}, x^{2}, x^{3}\right)\right\} \mid \sum_{i=1}^{3} x^{i} x^{i}<r^{\prime}\right\} \\
& \left.B_{2}^{\prime}=\left\{\left(\mathfrak{t}, x^{1}, x^{2}, x^{3}\right)\right\} \mid \sum_{i=1}^{3} x^{i} x^{i}<r^{\prime}\right\} \\
& \left.B_{3}^{\prime}=\left\{\left(x^{0}, x^{1}, x^{2}, x^{3}\right)\right\} \mid \sum_{i=1}^{3} x^{i} x^{i}=r^{\prime}, 0<x^{0}<\mathfrak{t}\right\} \\
& \left.C_{1}^{\prime}=\left\{\left(0, x^{1}, x^{2}, x^{3}\right)\right\} \mid \sum_{i=1}^{3} x^{i} x^{i}=r^{\prime}\right\} \\
& \left.C_{2}^{\prime}=\left\{\left(\mathfrak{t}, x^{1}, x^{2}, x^{3}\right)\right\} \mid \sum_{i=1}^{3} x^{i} x^{i}=r^{\prime}\right\},
\end{aligned}
$$


where $B_{3}^{\prime}$ is a timelike hypersurface and the other four are spacelike hypersurfaces. We define also the manifolds $B_{1} \subset B_{1}^{\prime}$ and $B_{2} \subset B_{2}^{\prime}$

$$
\begin{aligned}
& \left.B_{1}=\left\{\left(0, x^{1}, x^{2}, x^{3}\right)\right\} \mid \sum_{i=1}^{3} x^{i} x^{i}<r_{1}, r_{1}<<r^{\prime}\right\}, \\
& \left.B_{2}=\left\{\left(\mathfrak{t}, x^{1}, x^{2}, x^{3}\right)\right\} \mid \sum_{i=1}^{3} x^{i} x^{i}<r_{2}, r_{2}<<r^{\prime}\right\},
\end{aligned}
$$

which contain respectively ( see Figure 1 ) the field configurations $F(0, \mathbf{x})=$ $F_{1}(0, \mathbf{x})+F_{2}(0, \mathbf{x})$ and $F(\mathfrak{t}, \mathbf{x})=F_{1}(\mathfrak{t}, \mathbf{x})+F_{1}(\mathfrak{t}, \mathbf{x})$.

We denote the interior of $N$ by $U^{\prime}$ and also introduce the submanifold $U \subset U^{\prime}$ (Figure 1). Table 1 collects [22] the main features of the above submanifolds, necessary for the integrations (appearing in Stokes theorem) performed in the main text

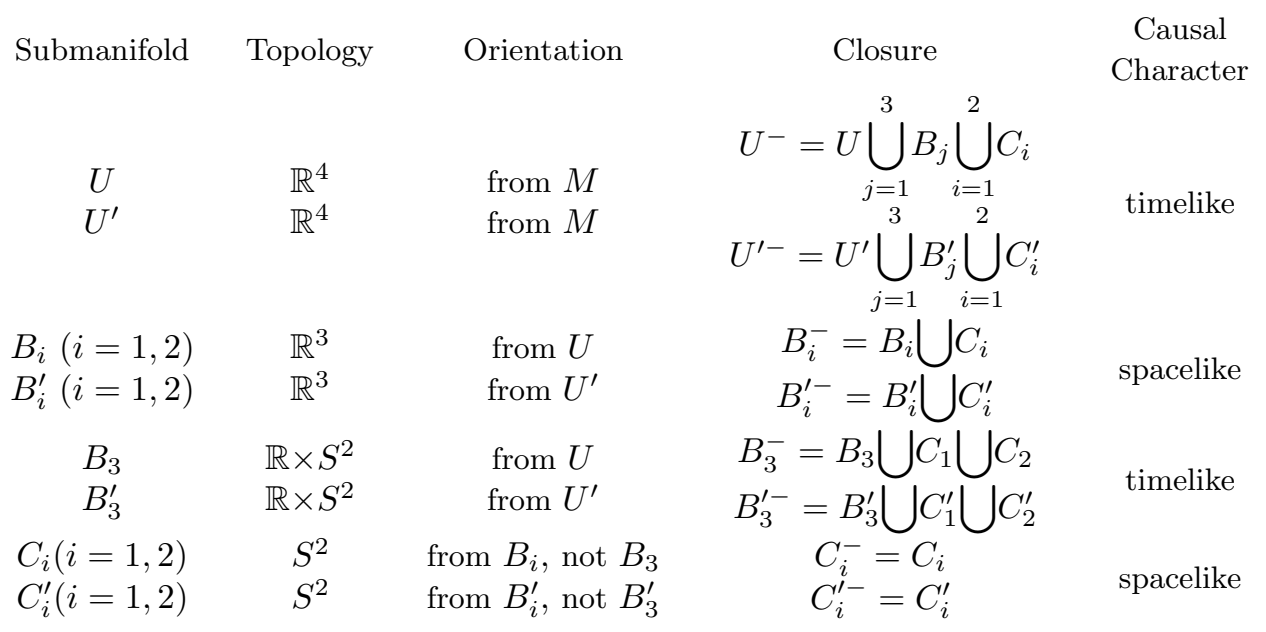

Table 1. Main Features of the Submanifolds $N, B_{i}^{\prime}, B_{i}, C_{i}^{\prime}$ and $C_{i}$

\section{References}

[1] Chen, F. C., Linearity of Electromagnetic Field Energy-Momentum, Am. J. Phys. 36, 390-393 (1968).

[2] Cornille, P., Advanced Electromagnetism and Vacuum Physics, World Scientific, Singapore, 2003.

[3] Farlow, S. J., Partial Differential Equations for Scientists and Engineers, J. Wiley \& Sons Inc., New York, 1982.

[4] Gauthier, N., What Happens to Energy and Momentum When Two Oppositely-Moving Wave Pulses Overlap?, Am. J. Phys. 71, 797-790 (2003). 
[5] Hestenes D;. Space Time Algebra, Gordon and Breach, New York, 1996.

[6] Jenkins, F. A. and White, H. E., Fundamentals of Physics Optics (4th edition), McGraw-Hill, New York, 1976.

[7] Kukhlevsky, S. V., Mechler, M., Csapó, L., Janssens, K. and Sameck, O., Enhanced Transmission Versus Localization of Light Pulse by a Subwavelenght Metal Slit, Phys. Rev. B 70, 195428 (2004). physics/0408013.

[8] Klein, M. V. and Furtak, T. E., Optics (2nd edition), J. Wiley \& Sons, New York, 1986.

[9] Kukhlevsky, S. V., Breaking of Energy Conservation Law: Creating and Destroying of Energy by Subwavelenght Nano Systems, physics/0610008.

[10] Kukhlevsky, S. V., Non Classical Energy-Momentum in Multi-wave Systems: "Extra-Energy", "Negative-Energy" and "Annihilation Energy", physics/0606055

[11] Levine, R. C., False Paradox of Superposition in Electric and Acoustic Waves, Am. J. Phys. 48, 28-31 (1980).

[12] Mathews, W. N., Superposition and Energy Conservation for Small Amplitude Mechanical Waves, Am. J. Phys. 54, 233-238 (1986).

[13] Mosna, R. A. and Rodrigues, W. A. Jr. ,The Bundles of Algebraic and Dirac-Hestenes Spinor Fields, J. Math. Phys 45, 2945-2966 (2004). math-ph/0212033.

[14] Notte-Cuello, E., da Rocha, R. and Rodrigues, W.A. Jr., The Effective Lorentzian and Teleparallel Spacetimes Generated by a Free Electromagnetic Field. gr-qc/0612098

[15] Parrot, S., Relativistic Electrodynamics and Differential Geometry, Springer-Verlag, New York, 1987.

[16] Rodrigues, W. A. Jr. , Algebraic and Dirac-Hestenes Spinors and Spinor Fields, J. Math. Physics 45, 2908-2944 (2004). math-ph/0212030

[17] Ribarič, M. and Šušteršič, L., Conservation Laws and Open Questions of Classical Electrodynamics, World Scientific, Singapore, 1990.

[18] Riez, M.., Clifford Numbers and Spinors: with Riesz's Private Lectures to E. Folke Bolinder and a Historical Review by Pertti Lounesto ,Fundamental Theories of Physics .54 ,Springer, Berlin,1993.

[19] Rodrigues, W. A. Jr. and Capelas de Oliveira, E., The Many Faces of Maxwell, Dirac and Einstein Equations. A Clifford Bundle Approach, RP 56/05 IMECC-UNICAMP, http://www.ime.unicamp.br/rel_pseq/2005/rp56-05.html 
[20] Rodrigues, W. A. Jr., Souza, Q. A. G., Rocha, R., Conservation Laws on Riemann-Cartan, Lorentzian and Teleparallel Spacetimes, math-ph/0605008]

[21] Rowland, D. R. and Pask, C., The Missing Wave Momentum Mystery, Am. J. Phys. 67, 378-388 (1999).

[22] Sachs, R. K. and Wu, H., General Relativity for Mathematicians, SpringerVerlag, New York, 1977.

[23] Thirring, W., Classical Field Theory, vol. 2, Springer-Verlag, New York, 1980.

[24] Strong, J., Concepts of Classical Optics, Fremann, San Francisco, 1958.

[25] Welti, R., Light Transmission Through Two Slits: The Young Experiment Revisited, J. Opt. A: Pure Appl. Opt. 8, 606-609 (2006).

[26] Wu, T.T. and Lehmann, Spreading of Electromagnetic Pulses, J. Appl. Phys. 58, 2064-2065 (1985). 


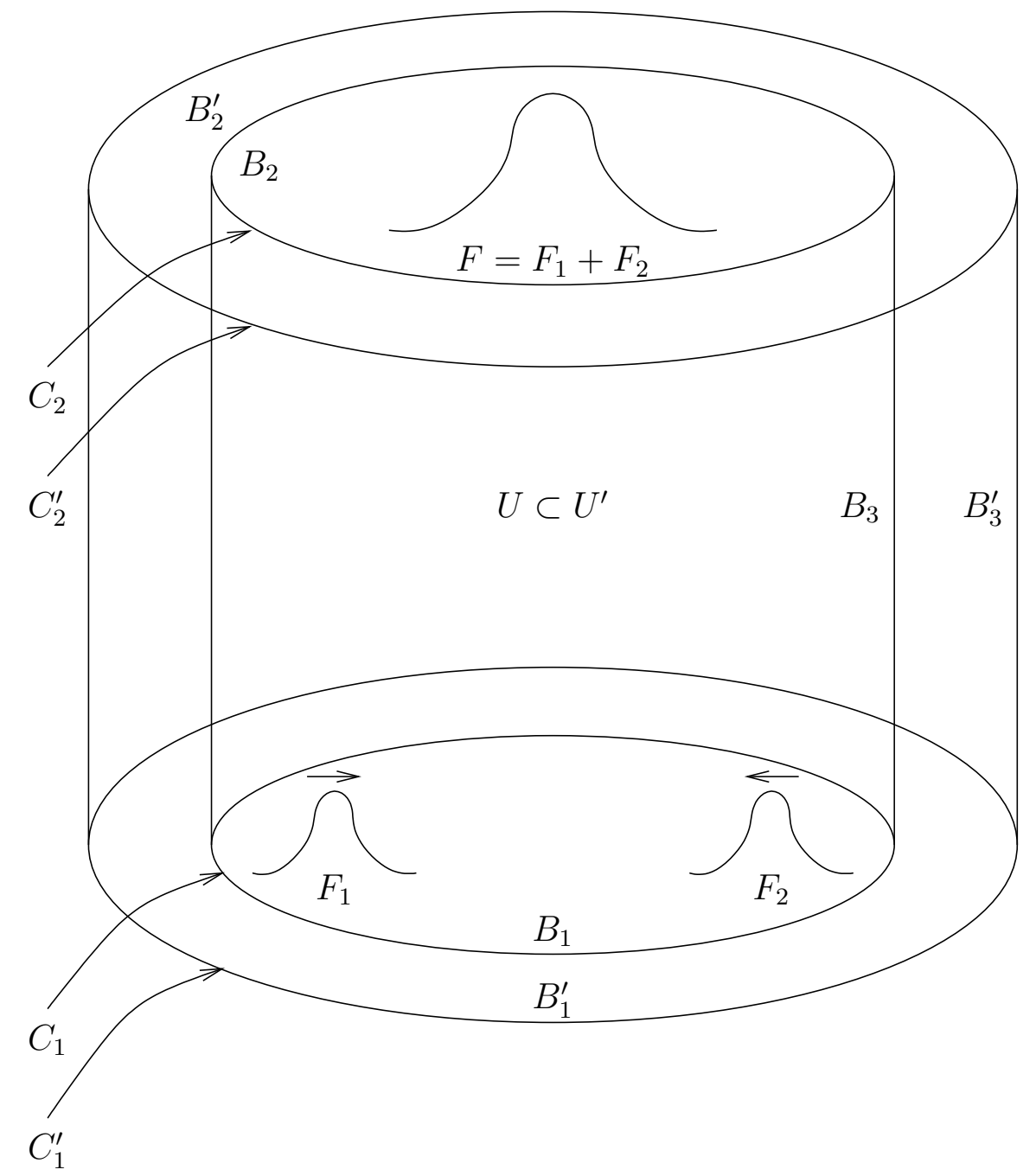

Figure 1: Standard Cylinder $N$ in Minkowski Spacetime, its Boundary Manifolds and the Field Configurations $F_{1}(0, \mathbf{x}), F_{2}(0, \mathbf{x})$ and $F(\mathfrak{t}, \mathbf{x})=F_{1}(\mathfrak{t}, \mathbf{x})+$ $F_{2}(\mathfrak{t}, \mathbf{x})$ 